\title{
Mathematical Model for Customer and Manufacturer Positions Coordination Procedure
}

\section{Gennadiy S. Rubin,} Yuliya V. Danilova and Marina A. Polyakova* Nosov Magnitogorsk State Technical University 38 Lenin, Magnitogorsk, 455000, Russia

Received 10.12.2014, received in revised form 23.01.2015, accepted 30.03.2015

The article presents a procedure formalization mathematical model for consumer demands and manufacturer capacities harmonization. It is proposed to divide the process into separate phases. At the first stage the choice of single product parameter area is carried out, based on which further work is fulfilled according to the target product functional oriented analysis. The next stage is to coordinate positions by selecting the optimum (compromise for the parties) product parameters level. Main approaches of qualimetry are used at this stage. Correspondent extent to each parameter is quantified within a set range. It is proposed to use quantitative assessment of customer requirements and manufacturer capabilities inconsistencies extent, and the matching process to describe in the form of curve. The developed mathematical model allows to formalize the conciliation procedure of customer demands and manufacturer capabilities.

Keywords: standardization, product, demands, customer, manufacturer, functional oriented analysis, qualimetry, mathematical model.

\section{Математическая модель}

\section{процедуры согласования позиций потребителя и изготовителя}

\author{
Г.Ш. Рубин, Ю.В. Данилова, М.А. Полякова \\ Магнитогорский государственный \\ технический университет им. Г.И. Носова \\ Россия, 455000, Магнитогорск, пр. Ленина, 38
}

Представлена математическая модель, позволяющая формализовать процедуру согласования требований потребителя продукиии и возможностей изготовителя. На первой стадии согласования позиций сторон выбирают единое пространство параметров

(C) Siberian Federal University. All rights reserved

* Corresponding author E-mail address: m.polyakova-64@mail.ru 
продукции, в котором происходит дальнейшая работа на основе функиионально-целевого анализа продукции. Следующий этап заключается в сближении позиций сторон путём выбора оптимального (компромиссного для сторон) уровня параметров продукиии. Данный подход позволяет формализовать процедуру согласования требований потребителя $u$ производителя исходя из оченки позищий обеих сторон.

Ключевые слова: стандартизация, продукиия, требования, потребитель, изготовитель, функционально-целевой анализ, квалиметрия, математическая модель.

\section{Введение}

В условиях рыночной экономики особую актуальность приобретают вопросы развития научных основ стандартизации, поскольку именно строгое соблюдение регламентированных правил и норм позволяет, с одной стороны, обеспечить безопасность продукции и услуг, а с другой - удовлетворить возрастающие потребности потребителя в новых видах продукции, повышении уровня предоставляемых услуг. В настоящее время разработка стандартов происходит на основе взаимного согласования мнений двух сторон - производителя и потребителя. Именно это во многом тормозит процесс разработки стандартов, увеличивает время их принятия, сдерживает внедрение результатов научных разработок в действующее промышленное производство.

\section{Постановка задачи исследования}

В работах $[1,2]$ сформулированы основные научные принципы разработки стандартов на промышленную продукцию, которые в совокупности составляют основу новой научной дисциплины - протипологии. Предметом данной науки, а также одной из ключевых процедур разработки промышленных стандартов является разработка методов согласования требований потребителя и возможностей изготовителя продукции. Нужно заметить, что требования потребителя в сочетании с возможностями изготовителя и в случае их совпадения дают возможность производства новой продукции, полностью удовлетворяющую обе стороны.

В настоящее время такое согласование сторон происходит в процессе переговоров, без использования специальных методов. В данной статье предпринята попытка формализовать процедуру согласования позиций потребителя и производителя.

\section{Метод решения}

Первой стадией согласования позиций сторон является выбор единого пространства параметров продукции, в котором происходит дальнейшая работа. Это достигается методами функционально-целевого анализа [3-6]. Следующий этап заключается в сближении позиций сторон путём выбора оптимального (компромиссного для сторон) уровня параметров продукции. В статье предложен механизм количественной оценки степени близости позиций сторон. Для этого мы предлагаем использовать аппарат квалиметрии, разработанный авторами Магнитогорской школы оценки качества $[4,5]$.

Степень согласования по каждому параметру оценивается количественно в диапазоне $[0,1]$, где 1 - оценка полного совпадения, 0 - расхождение, превышающее практически допустимое. Математическую модель количественной оценки разработаем по аналогии с методикой единичных оценок качества [5]. 
Обозначим через $p \geq 0$ абсолютную величину разности значений параметра, предлагаемых потребителем и изготовителем (иными словами, $p$ - мера расхождения между потребителем и изготовителем), через $M$ - оценку близости значений параметра. Тогда вблизи нуля, справа от него (рис. 1a)

$$
\mathrm{M}(p)=-k_{1} p^{2}+1, k_{1}>0 .
$$

Вблизи $p_{\max }$, слева от этого значения (рис. 1б)

$$
\mathrm{M}(p)=-k_{2}\left(p_{\max }-p\right)^{2}, k_{2}>0 .
$$

Назовем точки $(0,1),\left(p_{\max }, 0\right)$ реперными точками первого порядка.

Пусть $p_{b}$ - координата граничной точки участков кривой, прилегающих к реперным точкам первого порядка (реперная второго порядка). Тогда, учитывая (1), (2) и непрерывность изменения оценки, получим

$$
-k_{1} p_{b}^{2}+1=k_{2}\left(p_{\max }-p_{b}\right)^{2} .
$$

Кроме того, слева от рассматриваемой точки $\mathrm{M}^{\prime}(p)=-2 k_{1} p$, а справа $\mathrm{M}^{\prime}(p)=-2 k_{2}\left(p_{\max }-p\right)$. Используя дополнительно условие непрерывности скорости изменения оценки (первой производной), получаем систему уравнений

$$
\left\{\begin{array}{c}
-k_{1} p_{b}^{2}+1=k_{2}\left(p_{\max }-p_{b}\right)^{2} \\
k_{1} p_{b}=k_{2}\left(p_{\max }-p_{b}\right)
\end{array}\right.
$$

Второе уравнение системы даёт $k_{1}=k_{2} \frac{p_{\max }-p_{b}}{p_{b}}$. Подставляя это выражение для $k_{l}$ в первое уравнение системы, определим

$$
-k_{2}\left(p_{\max }-p_{b}\right) p_{b}+1=k_{2}\left(p_{\max }-p_{b}\right)^{2}
$$

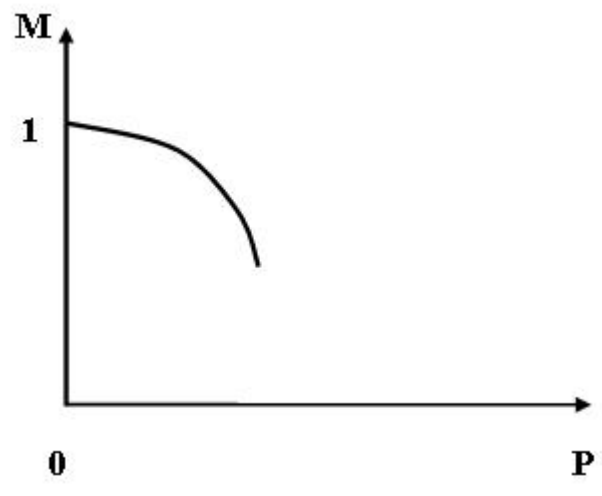

a

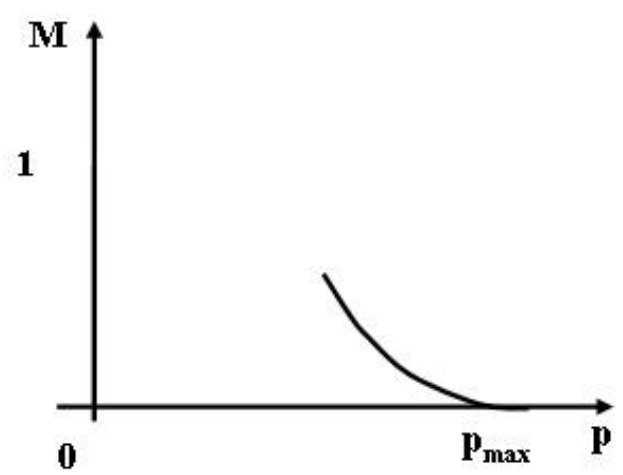

6

Рис. 1. Вид зависимости вблизи реперных точек первого порядка: а - участок кривой при максимальном расхождении позиций потребителя и изготовителя; б - участок кривой при сближении позиций потребителя и изготовителя 
Отсюда следующие формулы для нахождения $k_{1}$ и $k_{2}$ :

$$
\left.k_{2}=\frac{k_{1}=\frac{1}{p_{b} p_{\max }}}{p_{\max }\left(p_{\max }-p_{b}\right)}\right\} .
$$

Подставляя найденные выражения для $k_{1}$ и $k_{2}$ в формулы (1) и (2), получим:

$$
\begin{aligned}
& \text { если } 0 \leq p \leq p_{b}, \mathrm{TO} \mathrm{M}(p)=1-\frac{p^{2}}{p_{\max } p_{b}} ; \\
& \text { если } p_{b} \leq p \leq p_{\max } \text {, то } \mathrm{M}(p)=\frac{\left(p_{\max }-p\right)^{2}}{p_{\max }} \cdot \frac{1}{\left(p_{\max }-p_{b}\right)} .
\end{aligned}
$$

Тогда

$$
\mathrm{M}\left(p_{b}\right)=1-\frac{p_{b}}{p_{\max }}
$$

В частности, если $p_{b}=\frac{p_{\max }}{2}$ то $\mathrm{M}\left(p_{b}\right)=\frac{1}{2}$ и в целом если $p_{b}=k p_{\max } 0 \leq k \leq 1$, то $\mathrm{M}\left(p_{b}\right)=1-k$.

Следовательно, точка соединения двух участков графика зависимости оценки от показателя лежит (рис. 2) на прямой, соединяющей точки $(0,1),\left(p_{\max }, 0\right)$ и описываемой уравнением

$$
\mathrm{M}(p)=1-\frac{p}{p_{\max }} .
$$

\section{Обсуждение результатов}

При изложенном выше подходе конкретный вид кривой устанавливаем тремя реперными точками: двумя точками первого порядка и одной - второго. Это определяет следующий

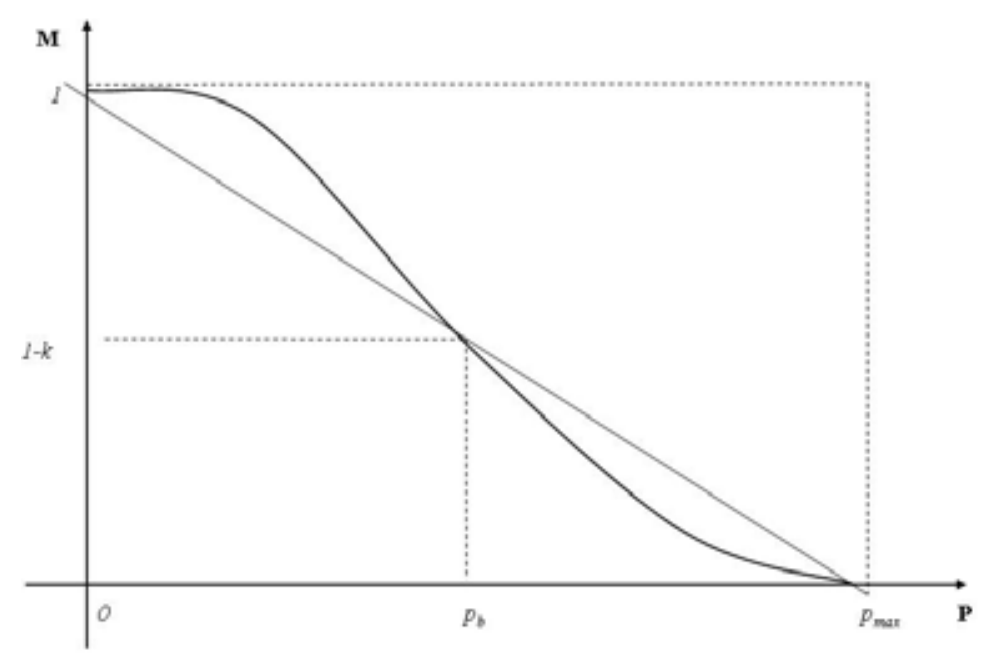

Рис. 2. Положение реперной точки второго порядка $p_{b}$

$$
-658-
$$


алгоритм построения кривой уравнения зависимости скорости изменения оценки от значения показателя:

1. $\mathrm{M}(0)=1$.

2. Находим $p_{\max }$ по условию $\mathrm{M}\left(p_{\max }\right)=0$.

3. Находим $p_{b}$ по условию $\mathrm{M}\left(p_{b}\right)=1-\frac{p_{b}}{p_{\max }}$.

4. При $0 \leq p \leq p_{b}$ определяем $\mathrm{M}(p)=1-\frac{p^{2}}{p_{\max } p_{b}}$,

5. При $p_{\max } \geq p \geq p_{b}$, определяем $\mathrm{M}(p)=\frac{\left(p_{\max }-p\right)^{2}}{p_{\max }\left(p_{\max }-p_{b}\right)}$.

6. При $p=0 \mathrm{M}(p)=1$ при $p \geq p_{\max } \mathrm{M}(p)=0$.

Если в качестве $p_{b}$ принять середину отрезка $\left[0, p_{\max }\right]$, т.е. $p_{b}=\frac{p_{\max }}{2}$, тогда

$$
\begin{aligned}
& \mathrm{M}(p)=1-\frac{2 p^{2}}{p_{\max }{ }^{2}}, \text { если } p \leq p_{b}, \\
& \mathrm{M}(p)=\frac{2\left(p_{\max }-p\right)^{2}}{p_{\max }^{2}}, \text { если } p \geq p_{b} .
\end{aligned}
$$

В общем случае при $0<k<1$ имеем

$$
p_{b}=k p_{\max } .
$$

Тогда

$$
\begin{aligned}
& \mathrm{M}(p)=1-\frac{p^{2}}{k p_{\max }^{2}} \text { при } p \geq p_{b}, \\
& \mathrm{M}(p)=\frac{\left(p_{\max }-p\right)^{2}}{p_{\max }^{2}(1-k)} \text { при } p \geq p_{b} .
\end{aligned}
$$

Итак, для определения зависимости необходимо знать реперные точки первого порядка. Эти точки определяются для любого вида зависимости оценки от расхождения показателей. Определение значения $p_{b}$ и оценки этого уровня показателя $1-k=\mathrm{M}\left(p_{b}\right)$ полностью формирует уравнение зависимости оценки от значения показателя. На практике для упрощения расчётов можно использовать линейную аппроксимацию полученной зависимости. Для получения линейной аппроксимации исследуем подробнее динамику изменения оценки. В интервале $\left(0, p_{b}\right)$ оценка убывает ускоренно с ускорением $k_{l}$, в интервале $\left(p_{b}, p_{\max }\right)$ оценка убывает замедленно с ускорением $k_{2}$. Таким образом, при значении показателя $p_{b}$ происходит переход от ускоренного убывания к замедленному. При этом в начале интервала $\left(0, p_{b}\right)$ скорость убывания меньше, чем равномерная на интервале (10), а во втором сегменте - больше. В начале интервала $\left(p_{b}, p_{\max }\right)$ скорость убывания больше средней равномерной, а во втором сегменте - меньше. Граничные точки этих сегментов - реперные третьего порядка $p_{a}$ и $p_{d}$, соответственно (рис. 3).

В этих точках касательные к графику зависимости $\mathrm{M}(p)$ (рис. 3, прямые II и III) параллельны прямой, проходящей через реперные точки первого и второго порядка (рис. 3, прямая I).

$$
-659-
$$




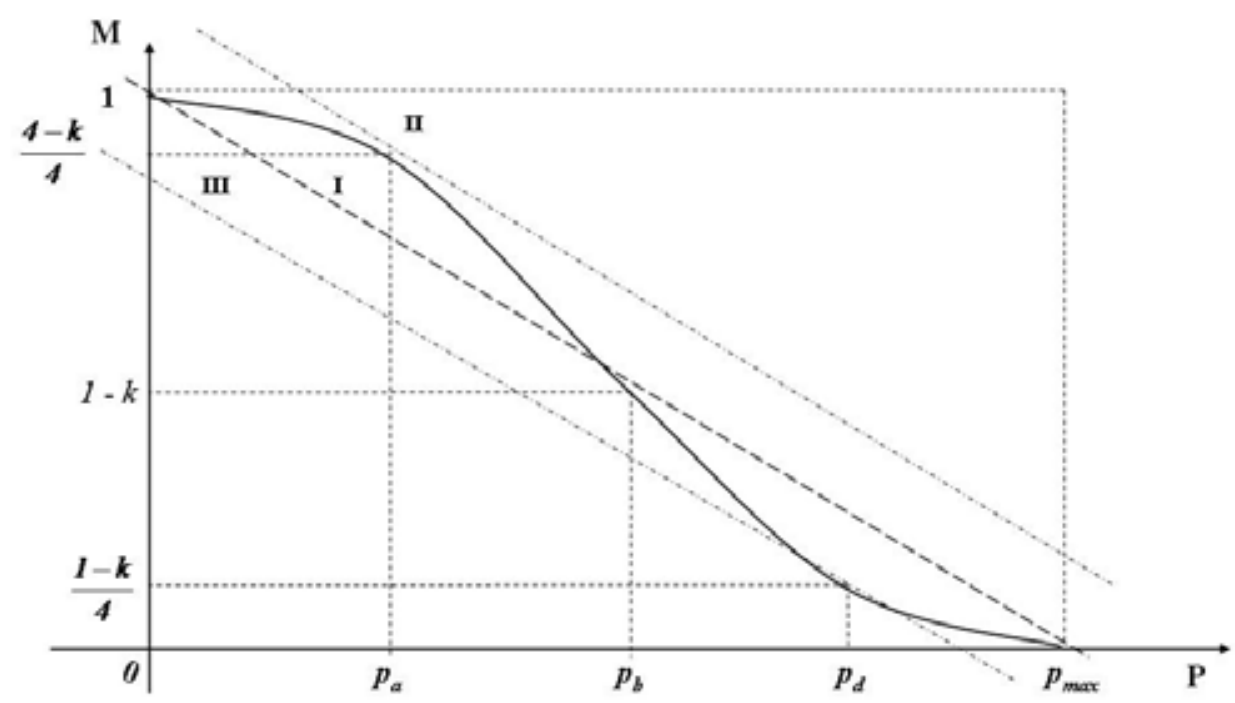

Рис. 3. Реперные точки третьего порядка

Тогда $\mathrm{M}^{\prime}\left(p_{a}\right)=\mathrm{M}^{\prime}\left(p_{d}\right)=-\frac{1}{p_{\max }}-$ угловой коэффициент прямой I и с учётом (7) и (8) $\mathrm{M}^{\prime}\left(p_{a}\right)=-\frac{2 p_{a}}{p_{\max } p_{b}}=-\frac{1}{p_{\max }} ; 2 p_{a} p_{\max }=p_{\max } p_{b}$

$$
\begin{gathered}
p_{a}=\frac{p_{b}}{2} ; \\
\mathrm{M}^{\prime}\left(p_{d}\right)=\frac{1}{p_{\max }} \cdot \frac{(-2)\left(p_{\max }-p_{d}\right)}{p_{\max }-p_{b}}=-\frac{1}{p_{\max }} ; \frac{2\left(p_{\max }-p_{d}\right)}{p_{\max }-p_{b}}=1 ; \\
p_{d}=\frac{p_{\max }+p_{b}}{2} .
\end{gathered}
$$

Из этого следует, что реперные точки третьего порядка являются серединами отрезков ускоренного и замедленного убывания, соответственно. Подставив выражения (14) в (7) и (15) в (8), получаем:

$$
\begin{gathered}
\mathrm{M}\left(p_{a}\right)=-\frac{p_{a}^{2}}{p_{\max } p_{b}}+1 ; \mathrm{M}\left(p_{a}\right)=-\frac{p_{b}^{2}}{4 p_{\max } p_{b}}+1=1-\frac{k}{4} ; \\
\mathrm{M}\left(p_{d}\right)=\frac{1}{p_{\max }} \cdot \frac{\left(p_{\max }-p_{d}\right)^{2}}{\left(p_{\max }-p_{b}\right)} ; \\
\mathrm{M}\left(p_{d}\right)=\frac{\left(p_{\max }-p_{b}\right)^{2}}{4 p_{\max }\left(p_{\max }-p_{b}\right)} ; \mathrm{M}\left(p_{d}\right)=\frac{1-k}{4}
\end{gathered}
$$

Итак, реперные точки третьего порядка определяются следующими формулами: 


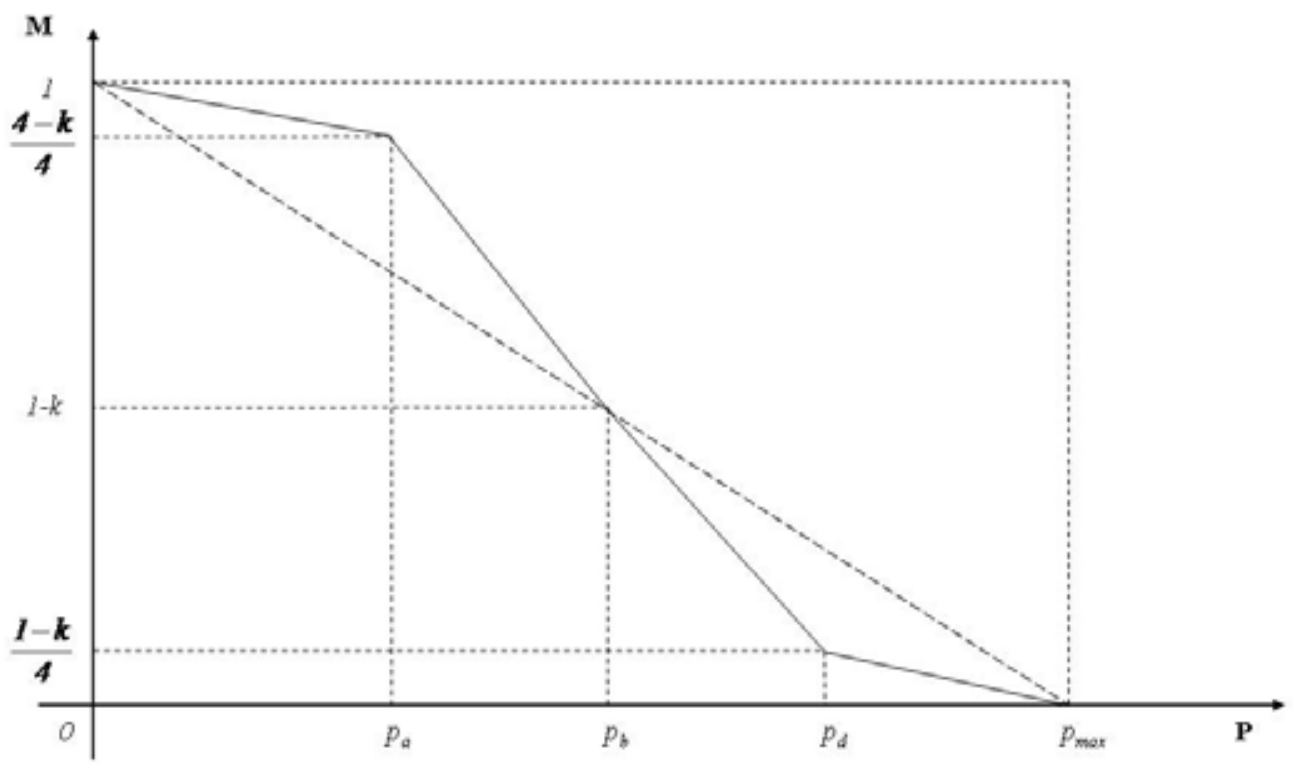

Рис. 4. Линейная аппроксимация зависимости оценки от показателя

$$
\begin{aligned}
& \mathrm{M}\left(p_{a}\right)=1-\frac{k}{4}=\frac{4-k}{4} ; \\
& \mathrm{M}\left(p_{d}\right)=\frac{1-k}{4} .
\end{aligned}
$$

По полученным координатам легко установить, что реперные точки второго и третьего порядков лежат на одной прямой. Линейную аппроксимацию зависимости М(р) построим как ломаную, соединяющую реперные точки. Таким образом, зависимость будет состоять из пяти отрезков прямой (рис. 4):

1. $\mathrm{M}(p)=1, p=0$.

2. $\mathrm{M}(p)=-\frac{1}{2 p_{\max }} p+1,0 \leq p \leq p_{a}$.

3. $\mathrm{M}(p)=-\frac{3}{2 p_{\max }} p+\frac{2+k}{2}, p_{a} \leq p \leq p_{d}$.

4. $\mathrm{M}(p)=-\frac{1}{2 p_{\max }} p+\frac{1}{2}, p_{d} \leq p \leq p_{\max }$.

5. $\mathrm{M}(p)=0, p \geq p_{\max }$.

Для комплексной оценки степени несоответствия требований потребителя и изготовителя мы предлагаем использовать деление показателей на доминирующие и компенсируемые [4]. Доминирующими считаются такие свойства, нулевая оценка которых приводит к нулевой комплексной оценке всех свойств данного уровня, а компенсируемыми - свойства, оценка которых повышает или понижает комплексную оценку свойств одного уровня, но не может обратить её в ноль.

Исходя из этого, комплексная оценка может быть получена [4]

$$
\text { - } 661-
$$




$$
C=\frac{\prod_{i=1}^{m} d_{i}^{1 / n}\left(d_{i}+1\right)^{\alpha_{i}} \prod_{i=1}^{n}\left(k_{i}+1\right)^{\beta_{i}}}{4},
$$

где $d_{i}$ - оценки доминирующих свойств, $i=1,2, \ldots, m, k_{i}$ - оценки компенсируемых свойств, $i=1,2, \ldots, n, \alpha_{i}$ и $\beta_{i}-$ весомости соответствующих свойств.

\section{Выводы}

Таким образом, разработанная математическая модель позволяет формализовать процедуру согласования требований потребителя и производителя исходя из оценки позиций обеих сторон. Это дает возможность оценить реальные сроки разработки стандартов, что будет способствовать их систематизации и упорядочению.

Работа выполнена в соответствии с госзаданием Министерства образования и науки Российской Федерации Магнитогорскому государственному техническому университету им. Г.И. Носова 11.1525.2014 «Разработка технологии получения высокопрочных длинномерных профилей из материалов с ультрамелкозернистой структурой в условиях комбинирования процессов интенсивного пластического деформирования».

\section{Список литературы}

[1] Рубин Г.Ш., Полякова М.А., Чукин М.В., Гун Г.С. // Сталь. 2013. № 10. С. 84-87.

[2] Рубин Г.Ш., Полякова М.А. // Вестник Магнитогорского государственного технического университета им. Г.И. Носова. 2014. № 1. С. 97-102.

[3] Рубин Г.Ш., Камалутдинов И.М. // Вестник Магнитогорского государственного технического университета им Г.И. Носова. 2010. № 1. С. 70.

[4] Рубин Г.Ш., Гун Г.С. // Деп. в ВИНИТИ. М., 1981. № 4105-8.

[5] Рубин Г.Ш. Квалиметрия метизного производства. Магнитогорск: ФГБОУ ВПО «МГТУ», 2012. $167 \mathrm{c}$.

[6] Рубин Г.Ш. // Вестник Магнитогорского государственного технического университета им. Г.И. Носова. 2011. № 2 (34). С. 29-30. 